htto://revistas.ucr.ac.crlindox.php/ingenieria www.ucr.ac.cr /ISSN 1409-2441

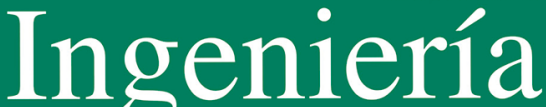

Revista de la Universidad de Costa Rica ENERO / JUNIO 2019 -VOLUMEN 29 (1)

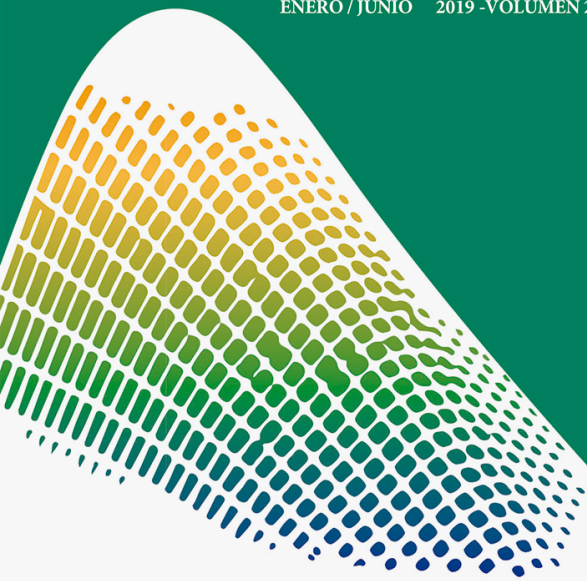




\title{
Deslizamientos en carreteras de montaña: propuesta de cálculo del bloque deslizante
}

\author{
Landslides on mountain roads: a calculation \\ proposal of the sliding block
}

\author{
Ingeniero Juan Antonio Picado Salvatierra, \\ MSc. Deslizamiento y estabilidad de taludes \\ Universidad de Costa Rica, San José, Costa Rica \\ juan.picado@ucr.ac.cr
}

\section{Recibido: 21 de agosto $2018 \quad$ Aceptado: 26 de setiembre 2018}

\begin{abstract}
Resumen
Para la determinación de posibles deslizamientos en los taludes que bordean las carreteras, sobre todo aquellas que atraviesan formaciones montañosas, de topografías de altas pendientes, se propone un método seudo estático, para suelos cohesivos puros, basado en la teoría de que ocurre una meteorización producto de la intemperización y de la humedad de la roca superficial de las montañas, y combinando las variables de pendiente, humedad, peso de la cobertura vegetal y del suelo, tanto seco como saturado, para así obtener valores que nos permitan predecir deslizamientos, y que permita mitigar la vulnerabilidad de las zonas susceptibles a ellos, lo que evitaría los accidentes, y un ahorro en tiempo y maquinaria.
\end{abstract}

Palabras clave:

Pendiente, humedad, cobertura vegetal, suelo, cohesión.

\begin{abstract}
For the determination of possible landslides on the slopes bordering roads, especially those that go through mountainous formations, of topographies of great slopes, a pseudo-static method is proposed, for purely cohesive soils, based on the theory that weathering occurs as a result of weathering and the humidity of the superficial rock of the mountains, combines the variables of slope, humidity, weight of the vegetal cover and of the soil. The investigation aims to obtain values that allow people to predict landslides. Moreover, this would allow to mitigate the vulnerability of the susceptible zones to them, what would prevent accidents, and a saving in time and machinery.
\end{abstract}

Keywords:

slope, humidity, vegetal cover, land, cohesion. 


\section{INTRODUCCIÓN}

Al ser Costa Rica un país montañoso, muchas de sus principales carreteras atraviesan topografías en las que predominan laderas de altas pendientes, lo que hace que continuamente ocurran deslizamientos que tienen grandes consecuencias, pues en muchos de los casos se deben hacer cierres de larga duración para poder limpiar las vías del material producto de ellas.

Con el fin de contar con una herramienta, con la que de una manera rápida, se pueda determinar mediante el cálculo de un Factor de Seguridad, la probabilidad de que determinado talud produzca un deslizamiento, se propone el método pseudo estático que se detalla en este documento; tomando en cuenta variables como pendiente, porcentaje de humedad, cohesión del suelo, peso del suelo, peso de la cobertura vegetal y la profundidad de la capa susceptible a deslizarse.

\section{METEORIZACIÓN}

La propuesta hecha en este artículo basa en gran parte su sustento, en que una gran cantidad de carreteras costarricenses atraviesan formaciones montañosas, en las que la meteorización hace que se forme una capa de suelo recubierta por una cobertura vegetal. De acuerdo con el Capítulo 12, "Meteorización y suelos", del libro de Juan María Montero Olarte geología, Universidad Nacional de Colombia (2003), se tiene lo siguiente:

Según una antigua concepción agronómica, en el suelo residual se reconocen tres horizontes: HORIZONTE A, donde las partículas disueltas en el proceso de descomposición, son removidas por las aguas de percolación hacia la parte inferior del perfil (lixiviación). Se denomina Horizonte Eluvial y es generalmente poroso. En la parte superior de este horizonte se concentra la materia orgánica donde se desarrolla la vegetación. HORIZONTE B, en el cual se acumula el material eluviado de la parte superior (Horizonte Iluvial) ; se caracteriza por ser algo cementado como consecuencia del efecto aglutinante del hierro y el aluminio removido en estado de solución o suspensión desde el horizonte superior. Debajo se sitúa el HORIZONTE C, que corresponde a la Roca Madre, es decir, la roca parental. Basadas todas ellas en el concepto agronómico descrito. (p. 263, 2003).

Como se puede inferir, en este caso los deslizamientos de las carreteras de montaña se producen cuando el componente del peso de la capa de suelo, (horizonte A), la hace deslizarse por ser mayor que su resistencia al esfuerzo cortante, que se produce entre la capa dura y la capa meteorizada, (horizontes A y B), ayudada por una alta pluviosidad que hace que la capa superficial se sature.

\section{COHESIÓN RESIDUAL}

La cobertura vegetal genera un peso adicional al peso del suelo, lo que induce a pensar que esto estaría haciendo que el talud del cual es parte, sea más propenso a deslizamientos. Por otro lado, hay que mencionar que las raíces de la vegetación dentro del 


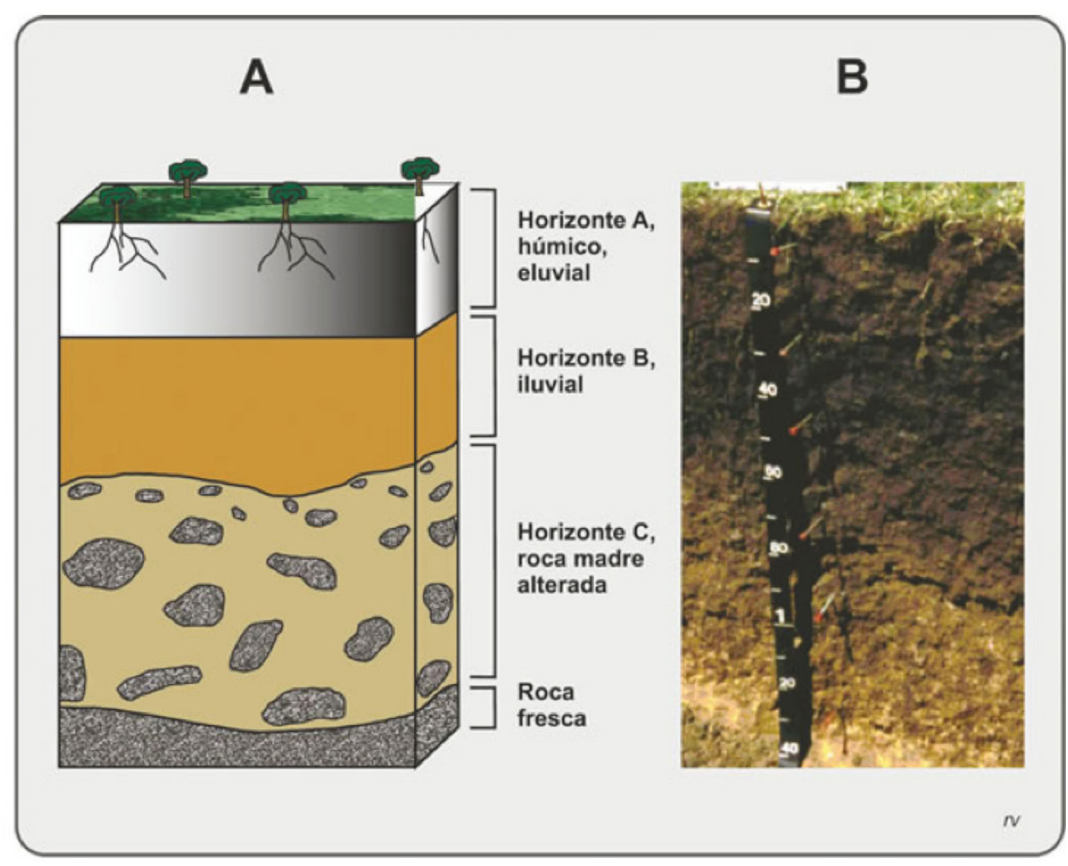

Figura 1. Modelo del bloque deslizante

(Tomado de http://www.insugeo.org.ar/libros/misc_21/10.htm)

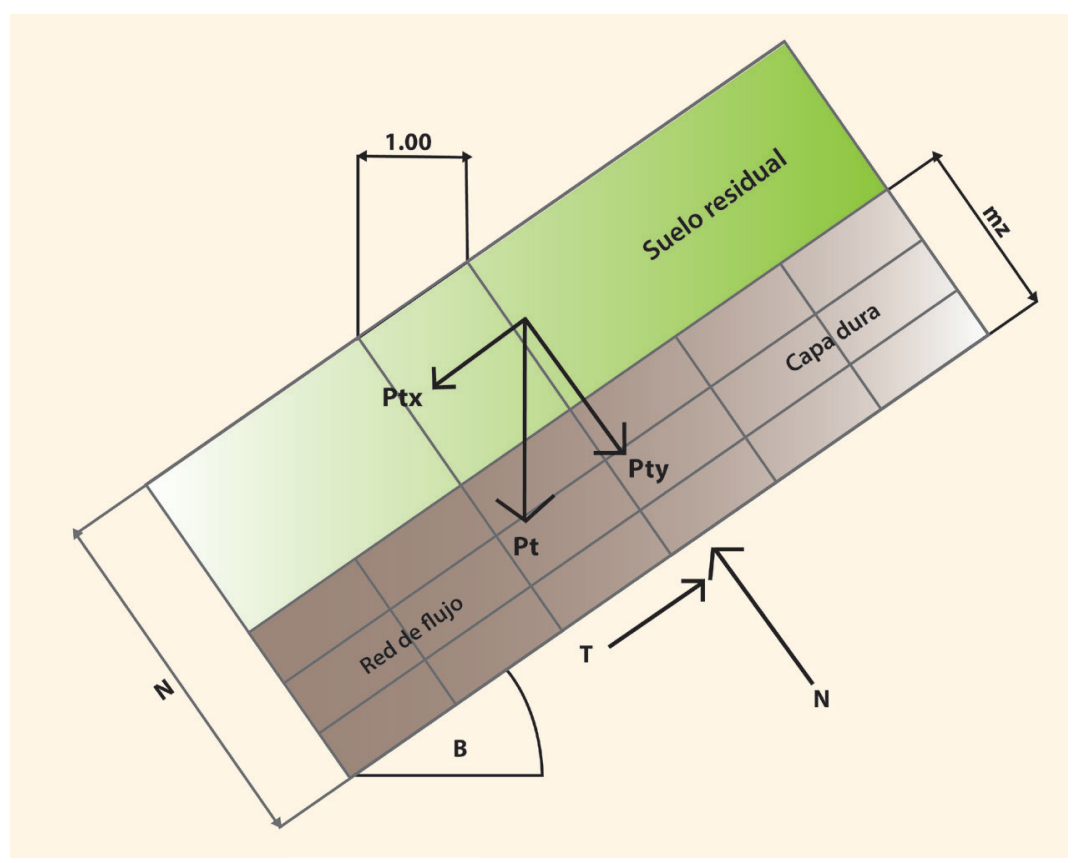

Figura 2. Modelo del bloque deslizante 
suelo, producen una resistencia al corte que muchos investigadores relacionan a la mecánica de ruptura del suelo, como un incremento a la cohesión del mismo, al oponer resistencia al movimiento de traslación del bloque de suelo sobre la superficie de ruptura que se forma en el límite entre esta y la capa dura debajo de ella.

\section{PESO DEL BLOQUE}

El peso de la masa del bloque está constituido por el peso del suelo y el de la cubierta vegetal sobre este, por lo que se debe de sumar el peso propio del suelo más el de la capa vegetal:

donde:

$$
P t=P s+P v
$$

$$
\begin{array}{ll}
\mathrm{Pt} & \text { peso total del bloque } \\
\mathrm{Ps} & \text { peso del suelo } \\
\mathrm{Pv} & \text { peso de la capa vegetal }
\end{array}
$$

Ahora bien, haciendo las siguientes relaciones, se llega a obtener el valor de los componentes del peso del suelo que actúa en favor del deslizamiento y la resistencia al esfuerzo cortante:

$$
\tau=P t x=P t * \text { seno } \beta
$$

donde:

$\tau \quad$ Resistencia al esfuerzo cortante del suelo.

Ptx Componente en el eje $\mathrm{x}$ del peso total.

$\mathrm{Pt} \quad$ Peso total del suelo

$\beta \quad$ Angulo del bloque deslizante respecto de la horizontal.

$$
N=P t y=P t * \cos \beta
$$

donde:

N Fuerza normal al plano de deslizamiento.

Pty Componente en el eje y del peso total.

Pt Peso total del suelo

$\beta \quad$ Angulo del bloque deslizante respecto de la horizontal.

El factor de seguridad estaría dado por:

$$
F=\frac{\text { Fuerzas resistentes al movimiento }}{\text { Fuerzas que propician movimiento }}
$$




\section{FACTOR M}

Este factor indica el porcentaje de saturación del suelo causado por la pluviosidad de la zona, y que está acotado entre 0 y 1 . El 0 indicará una saturación nula del suelo y 1 una saturación del 100\% del mismo.

\section{FUERZAS RESISTENTES AL MOVIMIENTO}

Se puede observar de la Figura. No. 2, que la fuerza que se opone al movimiento es la que ofrece la Resistencia al corte, dada por

$$
\tau=C u+\sigma * \operatorname{tg} \theta
$$

donde:

$\tau \quad$ Resistencia al esfuerzo cortante del suelo.

$\mathrm{Cu}$ Cohesión del suelo.

$\sigma \quad$ Resistencia al esfuerzo normal del suelo.

$\theta \quad$ Angulo de fricción interna del suelo.

Si tenemos suelos cohesivos, el ángulo de fricción interna sería igual a 0, por lo que la resistencia al esfuerzo cortante sería igual al valor de la cohesión:

$$
\boldsymbol{\tau}=\boldsymbol{C u}
$$

Ahora bien, numerosos estudios han encontrado que las raíces de la cubierta vegetal incrementan la cohesión del suelo, y por ende, la resistencia del mismo al esfuerzo cortante, por lo que este aumento es un valor que se sumaría a la cohesión que el suelo posea, y que se denominará Cohesión aportada por las raíces de la cubierta vegetal $(\mathrm{Cr})$, proponiéndose la siguiente fórmula para suelos cohesivos:

$$
\tau=C u+C r
$$

\section{FUERZAS QUE PROPICIAN EL MOVIMIENTO}

El componente del peso de la masa del suelo que hace que el bloque se deslice, es la fuerza que actúa a favor del deslizamiento, y en este caso, si se agrega el porcentaje de saturación del suelo, como un valor $\mathrm{m}, 0<\mathrm{m}<1$, permite obtener el peso del suelo como un total del porcentaje húmedo y seco que lo componen. Por tanto, pueden modelarse así las diferentes etapas por las que pasa en suelo, debido al tránsito de la época de lluvias, y la saturación que sucede producto de las mismas. 
Como la propuesta se refiere a un mecanismo de deslizamiento correspondiente al bloque deslizante el cual tiene un volumen, tomaremos una sección de un metro de ancho por un metro de fondo, quedándonos como una variable a determinar la profundidad (Z), Incorporando el valor de m en la ecuación anterior, se obtiene:

$$
\boldsymbol{P 1}=(\lfloor\boldsymbol{\gamma} * \boldsymbol{Z} * \boldsymbol{m}+\boldsymbol{\gamma} * \boldsymbol{Z} *(\mathbf{1}-\boldsymbol{m})\rfloor)
$$

donde:

P1 Peso parcial del bloque de suelo deslizante.

$\gamma$ s Peso del suelo saturado.

$\gamma \quad$ Peso del suelo.

$\mathrm{Z} \quad$ Profundidad del bloque deslizante.

m porcentaje de saturación del suelo.

Como se puede observar, en la ecuación anterior no se ha incorporado el peso de la Capa Vegetal como un elemento adicional al peso del bloque deslizante, con lo que el peso total de este quedaría:

$$
\boldsymbol{P t}=(\lfloor\gamma s * Z * \boldsymbol{m}+\gamma * Z *(\mathbf{1}-\boldsymbol{m})+\boldsymbol{P} \boldsymbol{v}\rfloor)
$$

donde:

Pt Peso total del bloque de suelo deslizante.

$\gamma$ s Peso del suelo saturado.

$\gamma \quad$ Peso del suelo.

$\mathrm{Z} \quad$ Profundidad del bloque deslizante.

m porcentaje de saturación del suelo.

Pv Peso de la capa vegetal.

Ahora bien, si se toma la fórmula (4), en donde se expresa el factor de seguridad $\mathrm{F}$ en términos de las fuerzas actuantes en contra y en pro del movimiento del bloque deslizante en suelos cohesivos, se tendría: 


$$
F=\frac{\tau}{P t x}
$$

Sustituyendo con fórmulas (2) y (5), se tendría:

$$
F=\frac{C u+C r}{\lfloor\gamma s * Z * m+\gamma * Z *(1-m)+P v\rfloor * \operatorname{seno} \beta}
$$

\section{PROPUESTA DE VALORES PARA LAS VARIABLES}

A continuación, se proponen valores para evaluarlos al introducirlos en las fórmulas que se proponen en este documento, tomando para ello el caso de los deslizamientos que se dan en la Ruta 32, en el tramo entre el Túnel Zurquí y el cruce de Río Frío. Lo anterior, a criterio personal, es en donde se dan la mayoría de deslizamientos de esta carretera.

\subsection{Peso de la cobertura vegetal}

De la Tabla 1: Estratos y fitomasa en Manaus, elaborada por Klinge y Rodríguez (1973), a su vez, tomada del libro: Producción Forestal para América Latina (Wadsworth, 2000), al existir ausencia en Costa Rica de valores similares, se pueden obtener datos del peso de la cobertura vegetal, de acuerdo con la altura de esta, los cuales se pueden utilizar para estimar el valor de la cobertura mencionada.

Analizando los datos de la Tabla 1, y luego de una comparación a priori de la altura de la cobertura en el tramo de la carretera de interés, o sea, el que atraviesa el Parque Braulio Carrillo, se propone utilizar una altura del estrato de 8 a 14 metros, siendo el peso de la cobertura que se indica por los autores del estudio de 77 toneladas por hectárea, lo que daría 0,008 toneladas por metro cuadrado.

\subsection{Cohesión aportada por las raíces de la cubierta vegetal $(\mathrm{Cr})$}

Se han hecho numerosos estudios respecto de este tema, tratando de determinar en todos ellos el valor probable del aumento de la cohesión debido a las raíces que la vegetación de la Capa Vegetal aporta.

En la siguiente figura 3, (Fariñas de Alba et al, 2002), el autor presenta los resultados de sus estudios, los cuales concluyen en que la acción de las raíces en el suelo, como se puede observar, incrementa la resistencia al corte del mismo, al comparar esta con un suelo sin cubierta vegetal.

Ahora bien, en la siguiente tabla tomada del libro Manual de estabilización y revegetación de taludes (Fariñas de Alba et al, 2002), se presentan diferentes valores 
Tabla 1. Estratos y fitomasa árborea en Manaus, Brasil.

\begin{tabular}{ccc}
\hline & \multicolumn{2}{c}{ Fitomasa fresca } \\
Altura del estrato $(\mathrm{m})$ & Peso medio (t/ha) & Porcentaje \\
\hline $24-35$ & 190 & 28 \\
$17-26$ & 400 & 58 \\
$8-14$ & 77 & 11 \\
$4-6$ & 16 & 2 \\
$2-3$ & 5 & 1 \\
Total & 688 & 100 \\
\hline
\end{tabular}

Klinge y Rodríguez (1973) en Wadsworth, 2000.

Tabla 2. Valores típicos del incremento en la cohesión del suelo debido a la acción de las raíces (Fariñas de Alba et al, 2002)

\begin{tabular}{llc}
\hline Autor & \multicolumn{1}{c}{ Suelo / Vegetación } & $\mathrm{C}_{\mathrm{g}}\left(\mathrm{kN} / \mathrm{m}^{2}\right)$ \\
\hline Swanston, (1970) & Coníferas de la montaña sobre morrenas & $3,4-4,4$ \\
O'Loughlin, (1974) & Coníferas de montaña sobre morrenas & $1-3$ \\
Endo y Tsurut, (1969) & Alisos de vivero en suelos de aluvión & $2,-12$ \\
Wu et al., (1970) & Coníferas de montaña sobre morrenas & 5,9 \\
Waldron et al.,(1981) & $\begin{array}{l}\text { Pinos de semillero cultivados en maceta } \\
\text { sobre suelos arcillosos }\end{array}$ & 5 \\
O`Loughlin y Zemer, (1982) & $\begin{array}{l}\text { Bosque perennifolio mixto sobre suelos } \\
\text { someros desarrollados en morreras }\end{array}$ & 3,3 \\
Gay y Megaham, (1981) & Coníferas en suelos arenosos & 10,3 \\
Riestenberg, Savonick y Dumford, (1983) & Arces en suelos de coluvión arcillosos \\
Borroughs y Thomas, (1977) & Coníferas en suelos de montaña & 5,7 \\
Barker y Hewlett & $\begin{array}{l}\text { Pastizal sembrado en bloques de hormigón } \\
\text { rellenos de suelo arcilloso reforzado con una } \\
\text { estructura celular }\end{array}$ & $3-17,5$ \\
\hline
\end{tabular}




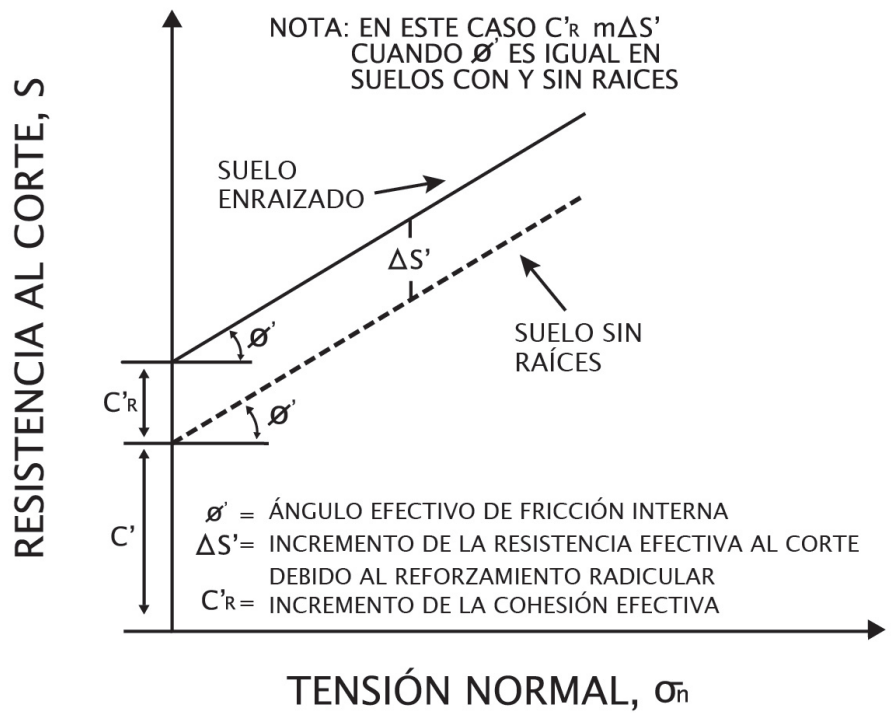

Figura 3. Efecto del reforzamiento radicular en la resistencia al corte del suelo.

(Fariñas de Alba et al, 2002)

\section{Factor de seguridad vs. porcentaje de saturación cohesión 1 ton $/ \mathrm{m}$}

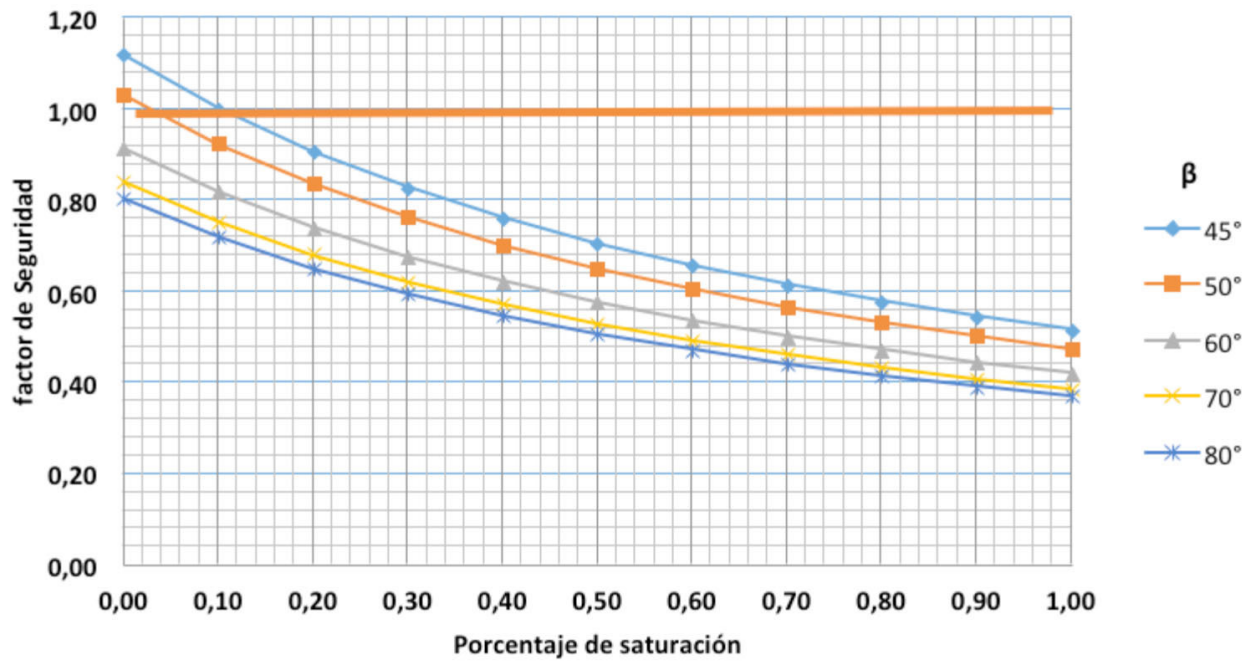

Gráfico 1. Factor de seguridad versus porcentaje de saturación. $\mathrm{Cr}=0,31 \mathrm{ton} / \mathrm{m}^{2}$, Cohesión del suelo 1 ton $/ \mathrm{m}^{2}$ 
resultado de la realización de estudios sobre el aumento de la cohesión en suelos causada por diferentes tipos de vegetación.

Como se puede observar, el tipo de vegetación estudiada no es la que se encuentra en las montañas costarricenses, por lo que se tendrá que hacer algún tipo de analogía, si se pretenden utilizar los valores que se indican.

Un valor crítico para la cohesión que las raíces producen, podría ser el de la cota inferior propuesto por Borroughsy Thomas en 1977, en la Tabla 2, para coníferas en suelos de montaña, $3 \mathrm{kN} / \mathrm{m}^{2}$, lo que equivaldría a $0.31 \mathrm{ton} / \mathrm{m}^{2}$, y a lo que su vez, daría factores de seguridad bajos, pues se incrementaría en poco la totalidad de la cohesión.

En el Gráfico 1, presentado a continuación, la cohesión del suelo usada es de 1 ton $/ \mathrm{m}^{2}$ (muy blando), la profundidad $\mathrm{Z}$ es de $2.00 \mathrm{~m}$, mostrando los resultados para algunos ángulos de talud, variando este de $45^{\circ}$ a $80^{\circ}$ con la vertical, un peso del suelo de 1,60 toneladas por metro cúbico y un peso saturado de 1,85 toneladas por metro cúbico. Además, se puede concluir que para los parámetros utilizados, los taludes serían inestables con solo un $10 \%$ de saturación, lo que se podría suponer que es un valor que fácilmente se alcanza al inicio de las épocas lluviosas, concluyéndose que es un talud con una gran probabilidad de generar un deslizamiento.

A su vez, se puede inferir que el valor de la cohesión del suelo de una tonelada es lo que determina que la resistencia sea baja, y en presencia de humedad, hace que los taludes se desestabilicen, produciendo deslizamientos.

Si se utiliza un valor de cohesión de 2,00 toneladas por metro cuadrado, y las mismas variables mencionadas, en el Gráfico 2 se puede notar cómo la resistencia a los deslizamientos aumenta considerablemente, pues se alcanza un Factor de Seguridad igual a uno con una humedad del $40 \%$ para pendientes del talud de $80^{\circ}$ con la horizontal. Nuevamente, al analizar los datos es posible deducir que la cohesión del suelo juega un papel protagónico en el desencadenamiento de deslizamientos.

\section{CONCLUSIONES Y RECOMENDACIONES}

- Para determinar la variable humedad del suelo será necesario contar con los registros pluviográficos de las estaciones meteorológicas cercanas al punto donde se quiera analizar. El régimen de lluvias es un proceso en constante cambio, tanto en su intensidad como en su periocidad, lo que hace necesario una actualización sistemática de los registros de ella.

- Es importante llevar un registro de los eventos de deslizamientos que se sucedan, con datos que podrían ser: coordenadas del lugar, hora, fecha, volumen aparente, dimensiones aparentes, tipo de cobertura, otros a determinar. Todo lo anterior, a través de un Sistema de Información Geográfico.

- Se puede verificar, a través de las fórmulas propuestas, que tanto la humedad del suelo como la cubierta vegetal, tienen un rol determinante en la estabilidad de los taludes.

- De ser posible, sería importante tener perforaciones que permitan conocer 
algunas variables tales como: profundidad y ancho de las capas del suelo, tipo de suelo, entre otros.

- De acuerdo a esta propuesta, se deberían de diseñar los taludes tomando en cuenta variables como humedad del suelo, cubierta vegetal, geometría adecuada, que permitan tener un grado de confianza aceptable sobre su estabilidad.

- Con el fin de implementar los insumos necesarios para aplicar las fórmulas, generar estudios que lleven a tener Tablas que muestren diferentes tipos de cubiertas vegetales, pesos promedios y cohesión adicional que puedan aportar, todo esto para nuestro medio.

\section{Factor de seguridad vs. porcentaje de saturación cohesión 2.00 ton $/ \mathrm{m}^{2}$}

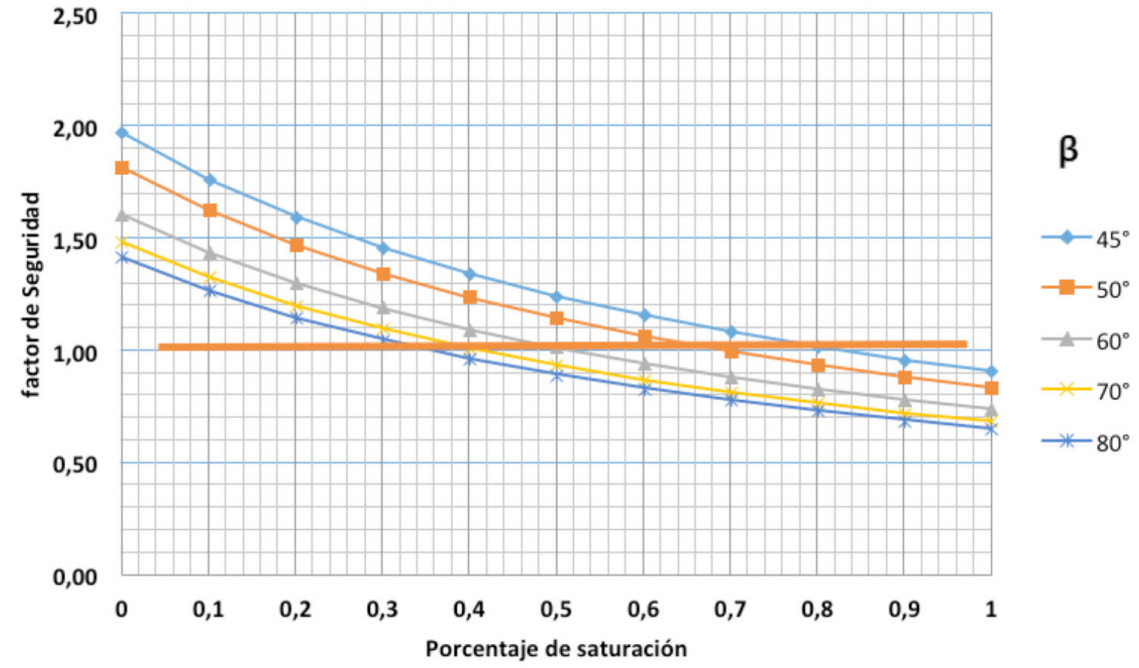

Gráfico 2. Factor de seguridad versus porcentaje de saturación. Cr=0,31 ton $/ \mathrm{m}^{2}$, Cohesión del suelo 2 ton $/ \mathrm{m}^{2}$.

\section{REFERENCIAS}

Craig, R. F. y Knapped, J. A. (2012). Craig's Soil Mechanics. London, Spon Press.

Fariñas de Alba, J. L., Gómez Prieto, R., Mataix González, C., García Bermúdez, P., Llopis Trillo, G., Serrano Pedraza, P. A., y López Jimeno, C. (2002). Manual de estabilización y revegetación de taludes. Madrid, Editorial Gráficas Arias Montano, S. A.

Meteorización y suelos (s.f.). In notas de Geología para Ingenieros Civiles y Ambientales. [archivo PDF] Recuperado de http://www.docentes.unal.edu.co/jmmonteroo/docs/12\%20 METSUELOS.pdf

Varela, R. (2014). Manual de geología. Recuperado de http://www.insugeo.org.ar/libros/ misc_21/10.htm).

Wadsworth. F. H. (2000). Producción foresta para América Latina. Recuperado de https://www. fs.fed.us/research/publications/producci\%F3n_forestal_para_am\%E9rica_tropical/cap.3.pd 


\section{ANEXOS}

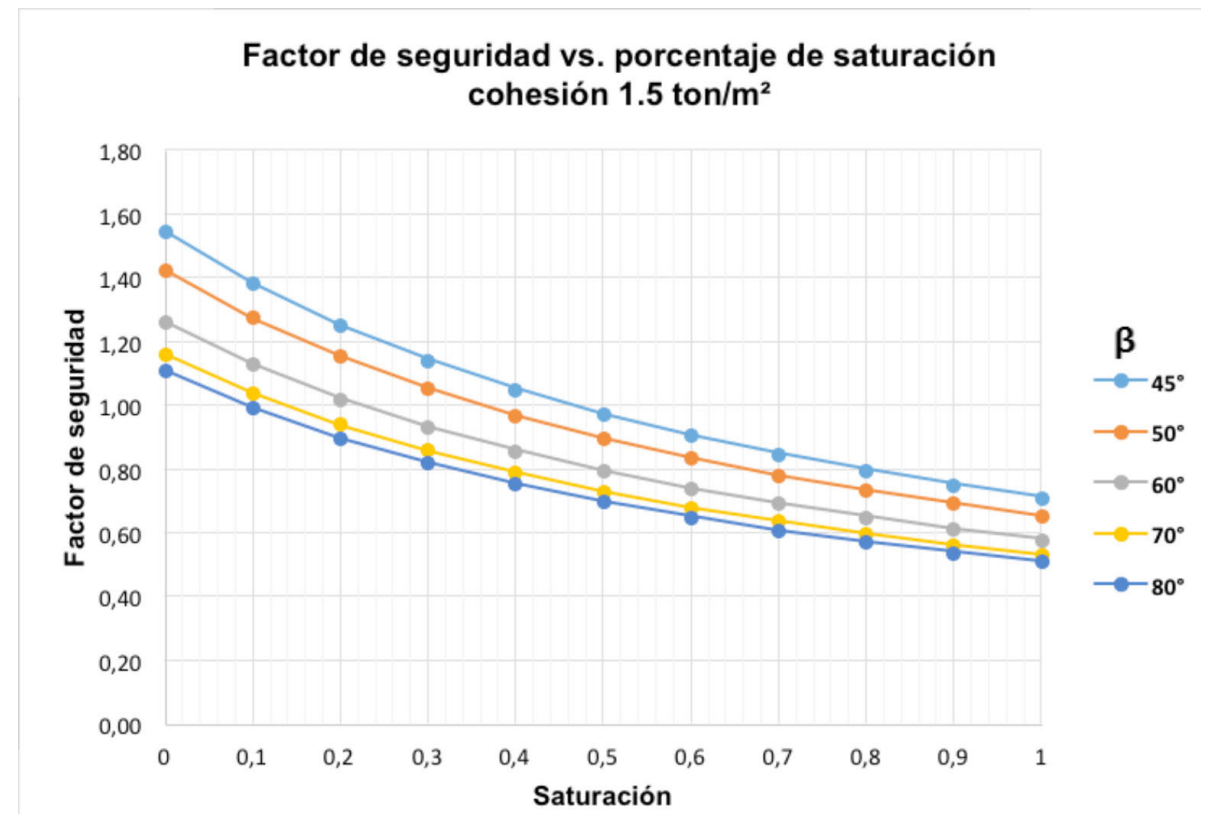

Gráfico 3. Factor de seguridad versus porcentaje de saturación. $\mathrm{Cr}=0,31$ ton $/ \mathrm{m}^{2}$, Cohesión del suelo 1,5 ton $/ \mathrm{m}^{2}$

Factor de seguridad vs. porcentaje de saturación Cohesión 2.00 ton $/ \mathrm{m}^{2}$

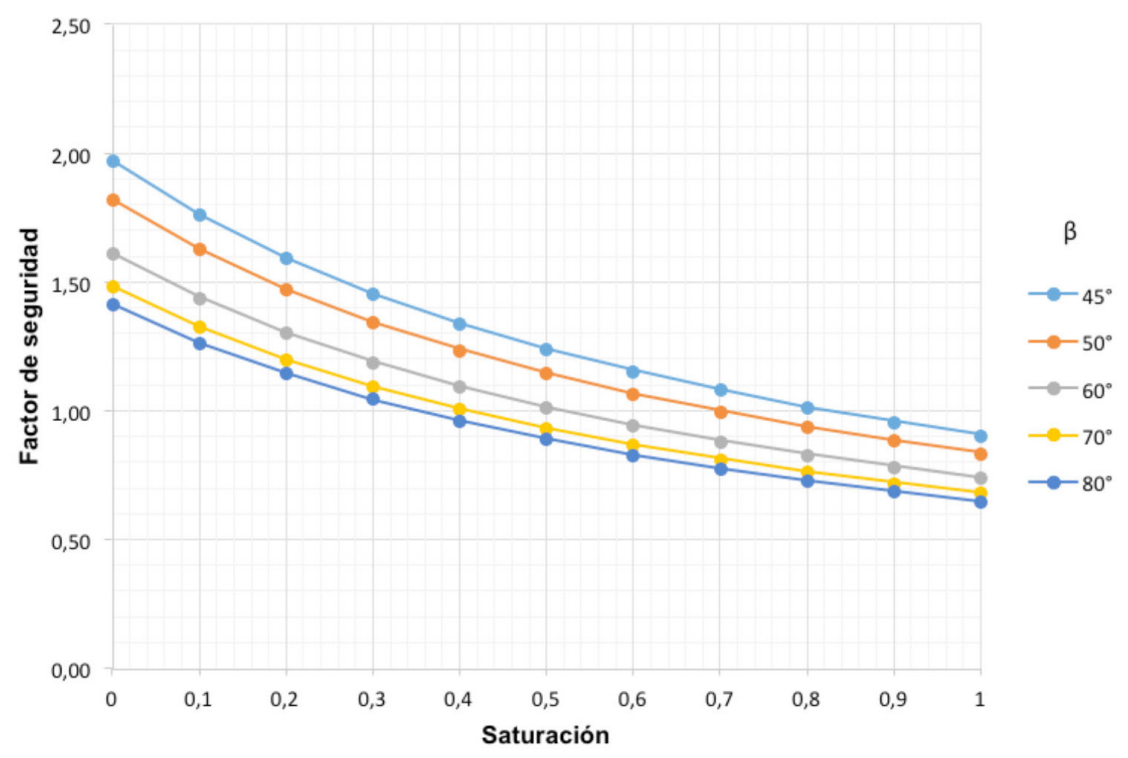

Gráfico No. 4: Factor de seguridad versus porcentaje de saturación. $\mathrm{Cr}=0,31$ ton $/ \mathrm{m}^{2}$, Cohesión del suelo 2,0 ton $/ \mathrm{m}^{2}$ 


\section{Factor de seguridad vs. porcentaje de saturación} Cohesión 2.50 ton $/ \mathrm{m}^{2}$

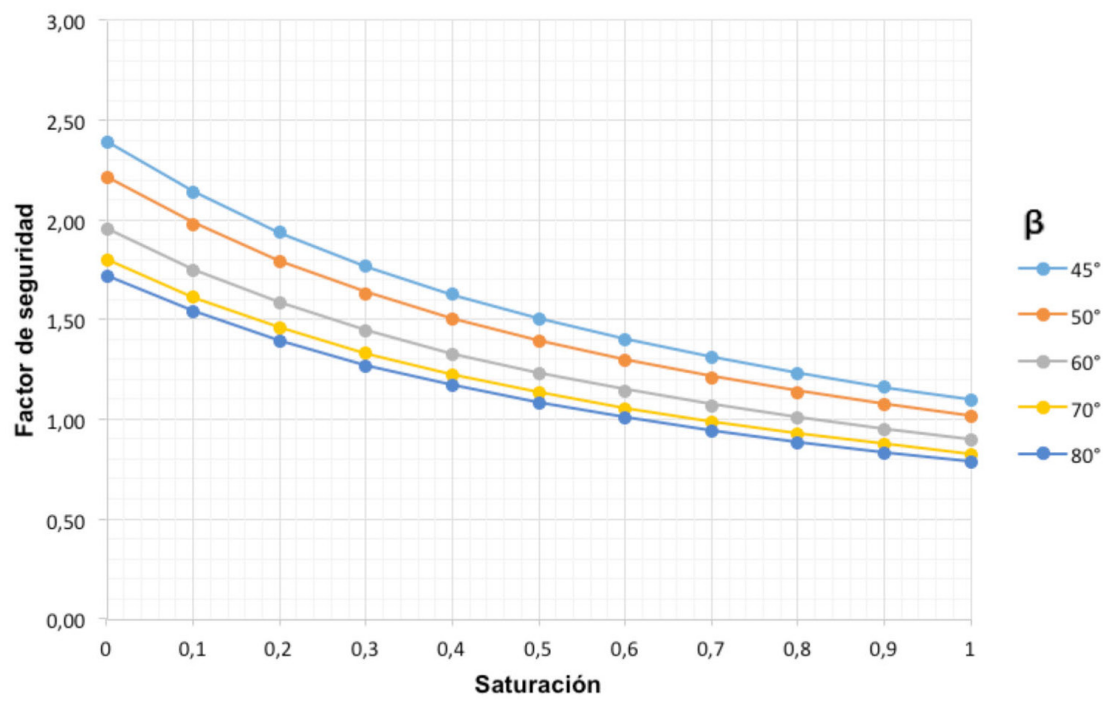

Gráfico 5. Factor de seguridad versus porcentaje de saturación. $\mathrm{Cr}=0,31$ ton $/ \mathrm{m}^{2}$, Cohesión del suelo $2,5 \mathrm{ton} / \mathrm{m}^{2}$ 